\title{
Linfoma histiocítico no epiteliotrópico cutáneo equino (Linfoma de células B alto en células T): Reporte de un caso clínico
}

\section{Equine Histiocytic Non-epitheliotropic Cutaneous Lymphoma (T-cell Rich B-Cell Lymphoma): Report of a Clinical Case}

\section{Linfoma histiocítico cutâneo equino não epiteliotrópico (Linfoma de celulas B rico em células T): relato de caso clínico}

Juan Estrada-McDermott ${ }^{2 \otimes}$, Miguel Somarriba-Soley ${ }^{3}$, Carla Murillo-Ramírez ${ }^{1}$, Javier Montero-Umaña ${ }^{4}$, José Vargas-Arrieta ${ }^{1}$, Manuel Estrada- Umaña ${ }^{1}$

1 Hospital Equino, Escuela de Medicina Veterinaria, Universidad Nacional, Costa Rica. carla.murillo.ramirez@una.cr, jose.vargas.arrieta@una.cr, juan.estrada.umana@una.cr

2 Estudiante de Postgrado, Escuela de Medicina Veterinaria, Colorado State University, USA. jmevet@colostate.edu

3 Estudiante de Postgrado, Scotland's Rural College (SRUC), Scotland, UK. somarvet@gmail.com

4 Médico Veterinario pensionado, Texas, USA.javmontero56@hotmail.com

Recibido: 27 de octubre de 2020 Corregido: 25 de marzo de 2021 Aceptado: 15 de abril de 2021

\section{Resumen}

Un equino adulto, raza Ibero, capón. con historia clínica de haber presentado "úlceras e inflamaciones por todo el cuerpo más pérdida crónica de condición” es atendido. El veterinario de la finca envió muestras para biopsia a un laboratorio privado de patología y refirió el paciente al Hospital Equino, Escuela de Medicina Veterinaria, Universidad Nacional, Costa Rica. Mientras el paciente estaba hospitalizado se reciben los resultados del patólogo privado con un diagnóstico de "linfoma histiocítico no epiteliotrópico cutáneo". El caballo es eutanasiado humanitariamente y el cuerpo examinado en el Departamento de Patología. La necropsia reporta los hallazgos macroscópicos \& microscópicos más relevantes confirmando el diagnóstico de "linfoma de células $\mathrm{B}$ alto en células $\mathrm{T}$ (linfoma histiolinfocítico cutáneo equino)”. Los resultados del examen histopatológico efectuado a varios órganos del paciente mostraron la presencia de un abundante infiltrado mononuclear compuesto de linfocitos, histiocitos y algunas células plasmáticas. Considerando que las patologías dermatológicas están entre los dos primeros grupos de enfermedades que afectan caballos es de gran importancia incluir esta enfermedad en el diagnóstico diferencial.

Palabras clave: Linfoma equino, células B, células T, histiolinfocítico, Costa Rica.

赵 Autor para correspondencia: jmevet@colostate.edu 


\begin{abstract}
An adult, Ibero breed, equine gelding, with a clinical history of "several ulcers and inflammations all over the body, plus a chronic loss of condition" is attended. The farm veterinarian sent biopsy samples to a private pathology laboratory and referred the horse to the Equine Hospital, School of Veterinary Medicine, at the Universidad Nacional de Costa Rica. While the patient was hospitalized, the results from the private pathologist were received, and a diagnosis of "equine histiocytic non-epitheliotropic cutaneous lymphoma" was made. The horse was humanely euthanized, and the body was examined at the Department of Pathology. Necropsy reports the most relevant macroscopic \& microscopic findings and confirms the diagnosis of "T-cell rich B-cell lymphoma (histiolymphocytic cutaneous lymphoma)." The histopathological results from examining several organs of the patient showed the presence of an abundant mononuclear infiltrate composed of lymphocytes, histiocytes, and some plasmatic cells. Considering that dermatological pathologies are amongst the first two groups of diseases affecting horses, it is of paramount importance to include this disease in the differential diagnosis.
\end{abstract}

Key words: equine lymphoma, B-cells, T-cells, histiolymphocytic, Costa Rica.

\title{
Resumo
}

Um equino adulto, raça Ibero, castrado, com uma história clínica de ter apresentado «úlceras e inflamaçóes por todo o corpo mais perda crônica da condição" é atendido. O veterinário da fazenda enviou amostras para biópsia a um laboratório particular de patologia e encaminhou o paciente ao Hospital Equino da Faculdade de Medicina Veterinária da Universidade Nacional da Costa Rica. Durante a internação do paciente, foi recebido o resultado do patologista particular com diagnóstico de "linfoma histiocítico cutâneo não epiteliotrópico". O sacrificio do animal foi realizado por meio de eutanásia humanitária e o corpo examinado no Departamento de Patologia. A necropsia relata os achados macroscópicos e microscópicos mais relevantes que confirmam o diagnóstico de "linfoma de células $T$ rico em células B (linfoma histiolinfocítico cutâneo equino)". O resultado do exame histopatológico de vários órgãos do paciente evidenciou a presença de abundante infiltrado mononuclear composto por linfócitos, histiócitos e algumas células plasmáticas. Considerando que as patologias dermatológicas estão entre os dois primeiros grupos de doenças que acometem cavalos, é de grande importância incluir esta doença no diagnóstico diferencial.

Palavras-chave: Linfoma eqüino, células B, células T, histiolinfocítico, Costa Rica.

\section{Introducción}

El linfoma (linfosarcoma) es una neoplasia maligna originada en linfocitos periféricos de varios tipos, en lo que se distingue de la leucemia que se origina en medula ósea (Muñoz et al., 2009), siendo la prevalencia en caballos más baja que en otros animales domésticos y afectando un amplio rango de edades sin predilección de género (Knottenbelt et al., 2015). El nombre de "Linfoma de células B alto en células $T$ " es propuesto por varios autores (Kelley et al., 1998; Scott et al., 2010; Valli et al., 2002; Valli 2007), aunque también se conoce como "linfoma histiocítico no epiteliotrópico cutáneo" o "linfoma histiolinfocítico cutáneo". Es una neoplasia bastante rara en caballos (0.6-3\% de los tumores de piel), donde hasta un $35 \%$ de los caballos afectados reportan lesiones cutáneas. En un estudio retrospectivo (1982-2010) efectuado en el Reino Unido se reporta una incidencia general de $14 \%$ para linfoma, con una disminución en las últimas décadas (Knowles et al., 2016). Mediante inmunohistoquímica se identifican once tumores de este tipo (Kelley et al., 1998; Scott et al., 2010). Histológicamente el linfoma se divide en epiteliotrópico y no epiteliotrópico. Los linfomas epiteliotrópicos son típicamente de linfocitos T, siendo asociado a leucemia y al Síndrome de Sézary, ambos subconjuntos del linfoma de células T. El linfoma no epiteliotrópico involucra dermis y subcutis (Scott et al., 2010). Miglio et al. (2019), describen los hallazgos clínicos e inmunofenotípicos para cuatro formas de 
linfoma equino. Bacci et al. (2020) mencionan características de un linfoma intestinal. Durham et al. (2013) reportan 203 casos de linfoma clasificados según el criterio de la Organización Mundial de la Salud. Se describe la clasificación y características clínicas en 88 casos de linfoma cutáneo equino (Miller et al., 2015). Se explica la caracterización inmunohistoquímica de receptores para estrógeno y progesterona en el linfoma de caballos (Henson et al., 2000). Clínica y morfológicamente el linfoma equino tiende a ser subdividido según su ubicación topográfica (Knottenbelt et al., 2015) siendo difícil determinar el sitio primario de la enfermedad pues las metástasis ocurren muy rápido y los pacientes son presentados al veterinario cuando ya tienen un estado avanzado. La literatura reporta (de Bruijn et al., 2007; Littlewood et al.,1995; Meisel et al., 2004; Meyer et al., 2006; Scott et al., 2010; Valli 2007) que varios órganos y sistemas pueden ser afectados, variando los signos según los órganos involucrados y duración de la enfermedad. La mayoría de los caballos tienen enfermedades sistémicas concurrentes, incluyendo pérdida de peso, letargia, linfoadenopatía, edema subcutáneo y pirexia, con progreso de semanas a meses. Estos pacientes pueden estar inmunosuprimidos, con anemia hemolítica inmunomediada, trombocitopenia o ambas (Scott et al., 2010). Las lesiones cutáneas son múltiples o extendidas, en forma de pápulas firmes y nódulos circunscritos $(0,5-2.0 \mathrm{~cm}$ de diámetro) con localización dermo-epitelial a subcutáneo, especialmente en párpados (unilateral o bilateralmente), donde su identificación es de importancia para un diagnóstico temprano (Murphy et al., 1989; Rebhun et al., 1998). Menos común son lesiones tipo urticaria, con placas grandes o áreas difusas de piel engrosada. Se puede encontrar prurito generalizado paraneoplásico y alopecia en caballos con linfoma difuso. Fenotipos histiolinfocíticos producen múltiples lesiones de piel antes de que el problema se haga sistémico y estas lesiones son subcutáneas, pudiendo ser difusas o agrupadas (Scott et al., 2010). Un linfoma con metástasis en los linfonodos mesentéricos fue asociado con intususcepción yeyunal (Matsuda et al., 2013). Existen comúnmente varios hallazgos de laboratorio clínico (Knottenbelt et al., 2015) que incluyen: citopenia de una o varias líneas (Ej. anemia), neutrofilia (leucocitosis), eritrocitosis, hipoalbuminemia, hiperglobulinemia, hipogammaglobulinemia con reducción o ausencia de $\operatorname{IgM}$ y susceptibilidad a infecciones secundarias, hipercalcemia en unos casos o hipocalcemia en otros e hiperfibrinogenemia. La medición de la timidina quinasa sérica es potencialmente un biomarcador útil para diagnosticar linfoma equino (Larsdotter et al., 2015). Las características histopatológicas e inmunofenotípicas del linfoma cutáneo maligno son descritas por de Bruijn et al. (2007). Histopatológicamente, el linfoma cutáneo equino presenta una infiltración, nodular a difusa, de la dermis profunda y la subcutis, algunas veces con proyecciones similares a dedos en el músculo esquelético subyacente. Las masas nodulares están compuestas por grandes linfocitos B neoplásicos acompañados por una población mayoritaria de pequeños linfocitos T reactivos con cromatina compacta densamente manchada y hendiduras nucleares poco profundas (Valli et al., 2002). Los linfocitos B forman hasta un $10 \%$ de la población total y tienen un gran núcleo vesicular con cromatina periférica y nucléolos prominentes más figuras mitóticas a menudo atípicas. Una tercera población celular se presenta ocasionalmente con macrófagos epiteloides y células gigantes histiocíticas multinucleadas. En esta neoplasia un aspirado ganglionar establece el diagnóstico tentativo, pero no debe reemplazar a un examen histológico completo por biopsia. Desde 1994 se recomienda el uso diagnóstico de ultrasonido (Garber et al., 1994). Se reporta el uso de biopsia guiada por ultrasonido en un caso de linfosarcoma mediastinal (de Clercq et al., 2004). Para la confirmación laboratorial del diagnóstico algunos autores consideran recomendable realizar inmunohistoquímica (Kelley et al., 1998; Littlewood et al., 1995; Scott et al., 2010). En un caso de linfoma gastrointestinal equino se usó una técnica diagnóstica de inmunocitoquímica en fluido peritoneal (Duran et al., 2016). Se reporta que ocurre una completa remisión del linfoma al inyectarse los nódulos con 
glucocorticoides y administrarse progestágenos orales (Littlewood et al., 1995). Los corticoides pretenden reducir el tamaño de las masas, pues el linfoma genera una considerable respuesta inflamatoria caracterizada por flujo celular hacia el tumor, aumentando su tamaño. Suprimir la inflamación con esteroides disminuye el tamaño del linfoma, haciéndolo mejor candidato para remoción quirúrgica, cuando aplica, o quimioterapia, además de reducir presión sobre órganos vecinos. El tiempo de supervivencia promedio (de Bruijn et al., 2007) después del descubrimiento de los primeros nódulos subcutáneos fue 3.8 años (rango 2.5 años: $\mathrm{n}=4$ ).

\section{Diagnóstico y manejo del caso clínico}

\section{Historia Clínica}

Un paciente de la especie equina, macho castrado, con 10 años de edad, raza Iberoamericana, con gran valor sentimental para los propietarios pues lo usan esporádicamente para Equinoterapia y procedente de la provincia de Limón, Costa Rica, ha sido afectado en los últimos meses por úlceras e inflamaciones por todo el cuerpo más pérdida crónica de condición. El Médico Veterinario de la finca: a) Toma biopsias por excisión de ulceraciones localizadas plantares a las cuartillas, además de muestras de nódulos subcutáneos ubicados distalmente a tarsos y cerca de la tuberosidad coxal, b) Envía dichas muestras a un laboratorio privado de Patología, y c) Refiere el paciente al Hospital Equino, Escuela de Medicina Veterinaria, Universidad Nacional, Costa Rica. El examen clínico del caballo internado muestra: 1) Lesiones ulcerosas en varios sitios de piel, ollares y meatos respiratorios con secreciones sero-sanguinolentas bilaterales provenientes de respiratorio, 2) Bloqueo del ducto nasolacrimal debido a las lesiones de piel, 3) Membranas mucosas muy pálidas sugestivas de anemia, 4) Edemas ventrales en extremidades, prepucio y escroto, 5) Nódulos subcutáneos en varias locaciones y 6) Pérdida de condición física (Ver Fig. 1), además de 7) Tos ocasional a pesar de que la auscultación de pulmones y bronquios mostró severa congestión y 8) Temperatura corporal alrededor de los $38^{\circ} \mathrm{C}$ aunque ocasionalmente presentaba picos de pirexia relativa con $1^{\circ} \mathrm{C}$ más. Se consideró que las complicaciones bacterianas secundarias en lesiones de las extremidades podrían haber influido en el prurito con automutilación mostrado en las mismas. En este momento el laboratorio privado de Patología reporta "Linfoma histiocítico no epiteliotrópico cutáneo”. En el informe se describe que "en toda la dermis hay una completa invasión de dos tipos de células mononucleares ambas con poco citoplasma visible, unas son más pequeñas con núcleo redondo-ovalado basofílico. Las otras son más grandes y pálidas con varias formas, algunas arriñonadas”.

En vista de los hallazgos clínicos y el reporte citado, se efectúa la eutanasia humanitaria del paciente y el cuerpo es enviado al Laboratorio de Patología, Escuela de Medicina Veterinaria, Universidad Nacional, Costa Rica, para su análisis.

\section{Diagnóstico}

Se envía hisopado de las lesiones nasales al Laboratorio de Bacteriología, misma Escuela de Medicina Veterinaria, que reporta un Streptococcus equi $(4+)$ sensible a Ceftiofur, Eritromicina, Tetraciclina, Penicilina y resistente a Trimetropin Sulfa. Otra muestra enviada a un Laboratorio de Micología privado arroja resultados negativos por hongos. La confirmación del diagnóstico final de linfoma se hace por necropsia con la respectiva histopatología (hallazgos adjuntos). 


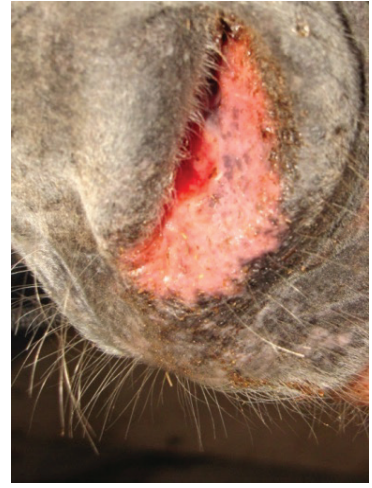

(a)

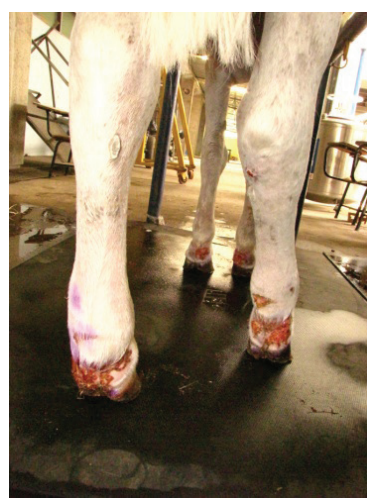

(d)

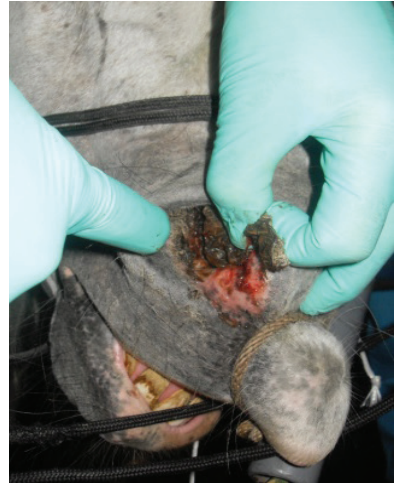

(b)

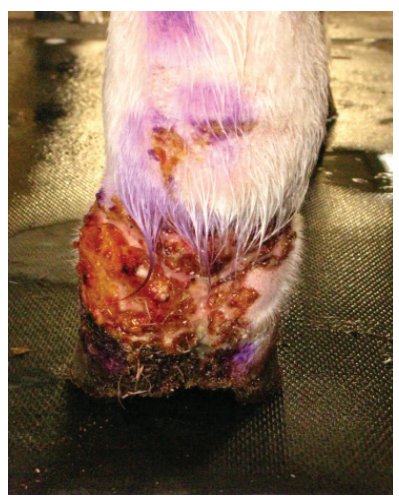

(e)

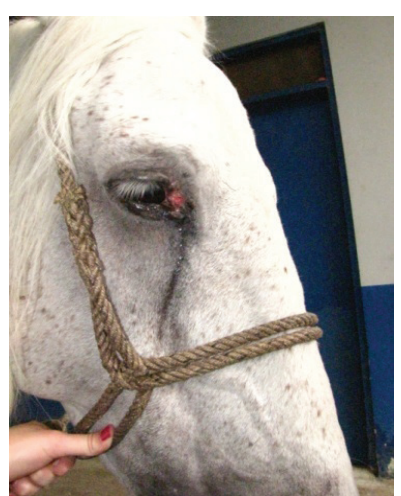

(c)

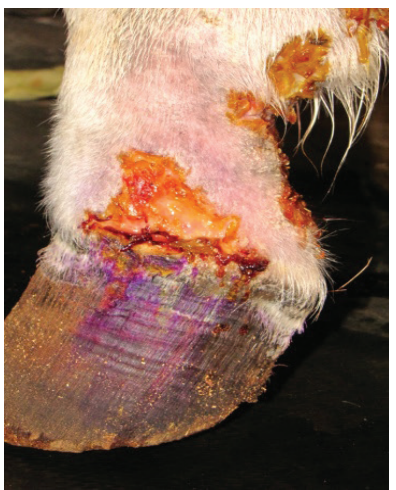

(f)

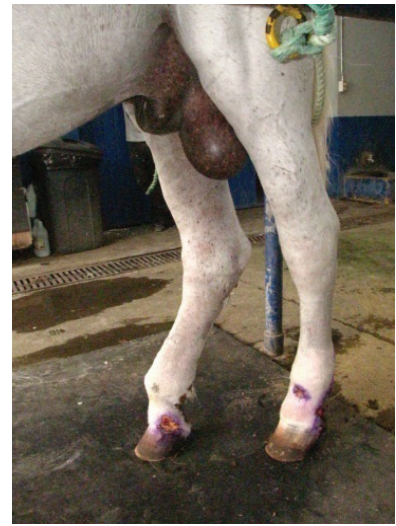

(h)

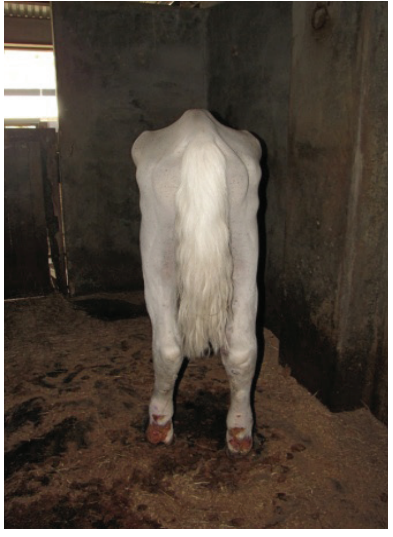

(i)

Figura 1 - Galeria de fotografias: "Signos clinicos del linfoma equino". Donde: (a), (b) y (c) muestran úlceras en mucosas de meato nasal y la comisura anterior del ojo, que bloquean el pasaje de lágrimas al ducto nasolacrimal, causando el consiguiente derrame sobre la cara; (d) y (e) presentan edema distal de patas con varias áreas de ulceraciones profundas en piel; en el caso de (f) vemos lesiones afectando severamente la banda coronaria y la calidad de la pared del casco; en (g) se aprecia edema ventral de prepucio, escroto y extremidades distales y en (b) vemos algunos nódulos subcutáneos hacia pelvis además de un marcado deterioro corporal que se agravó con el paso del tiempo. Fotografías por los autores. 


\section{Reporte de Necropsia}

El informe (Caso No. 47-11), Cátedra de Patología, Escuela de Medicina Veterinaria, UNA, reporta lo siguiente. Macroscópicamente se observa una "condición corporal baja”, "a nivel distal de los cuatro miembros lesiones ulceradas con costras y edema moderado", "fosas nasales con presencia de gran cantidad de úlceras con formación de costras serosanguinolentas", "al abrir la cavidad torácica se observa poca cantidad de líquido serosanguinolento" y "el corazón presenta hidropericardio leve”, "los pulmones muestran una coloración rojizo-azulada difusa bilateral con severa bronconeumonía purulenta, en algunos puntos abscedada, con zonas de necrosis", "los linfonodos bronquiales y mediastínicos presentan aumento de tamaño, algunos con material purulento en su interior, incluso absceda", "hígado y bazo presentan una congestión moderada", "los riñones muestran zonas decoloradas en la corteza", "la vejiga urinaria presenta acumulo de fibrina y material purulento en su interior" y "a nivel gastrointestinal se observa un aumento en varios linfonodos mesentéricos, algunos abscedados y lesiones de hemomelasma en la serosa del intestino delgado”. En concordancia con la clínica, el informe patológico reportó rinitis crónica mixta y una severa bronconeumonía purulenta necrotizante abscedada. A nivel histopatológico, tinción de Hematoxilina y Eosina (H\&E), se muestran los hallazgos más relevantes del examen. Ver Fig. 2.

\section{Discusión}

El linfoma cutáneo es infrecuente en la especie equina y la biopsia por excisión quirúrgica usada es el método de elección para el correcto diagnóstico histopatológico, aunque para confirmarlo se podría usar inmunohistoquímica (Scott et al., 2010). El paciente atendido concuerda con los grupos de edad descritos por la literatura (Knottenbelt et al., 2015). Los nódulos cutáneos (Littlewood et al., 1995) son típicos de este linfoma, aunque en el paciente aquí discutido predominaron las lesiones ulcerativas. Como reportado (Scott et al., 2010; Valli 2007), en este caso se pudo observar la proliferación de linfocitos e histiocitos pleomórficos que podrían ser células gigantes, binucleadas o multinucleadas. Aunque se sabe que la forma histiolinfocítica puede estar latente por años antes de manifestarse clínicamente, el prognóstico para linfoma generalmente es grave y los pacientes mueren o son eutanasiados pues los tratamientos no reportan en general buenos resultados (Scott et al., 2010). El uso de quimioterapia en el tratamiento del linfoma equino es limitado, caro y no accesible en Costa Rica. Según Scott et al. (2010) hasta ese año no hay publicaciones al respecto. Sin embargo, Luethy et al., en el año 2019 publican un estudio retrospectivo (1991-2017) de la respuesta del linfoma a quimoterapia en 20 caballos reportando los resultados obtenidos. El prognóstico para un caballo afectado con esta enfermedad depende del tipo de linfoma y del estado físico como el animal llega al veterinario, pero la muerte es el resultado común (de Bruijn et al., 2007), siendo la excisión quirúrgica de la masa, radiación y quimoterapia generalmente paliativos (Taintor et al., 2011). Algunos estudios (Henson et al., 2000; Scott et al., 2010) mencionan que el 58\% de los linfomas de "células B alto en células T" poseen receptores de progesterona, siendo los linfocitos B los que más expresan dichos receptores, por lo que el tumor da positivo a tinción. Un pequeño porcentaje de linfocitos del tejido normal se muestran positivos, sugiriendo que los linfocitos normales equinos pueden expresar receptores de progesterona (Henson et al., 2000). Estudios ultrasonográficos y radiográficos pueden reportar hallazgos relevantes tales como masas con imagen multilobar y homogéneamente hipoecoicas con áreas de necrosis, siendo la efusión pleural un hallazgo común en caballos con linfosarcoma mediastinal y el análisis citológico del fluido pleural importante en 


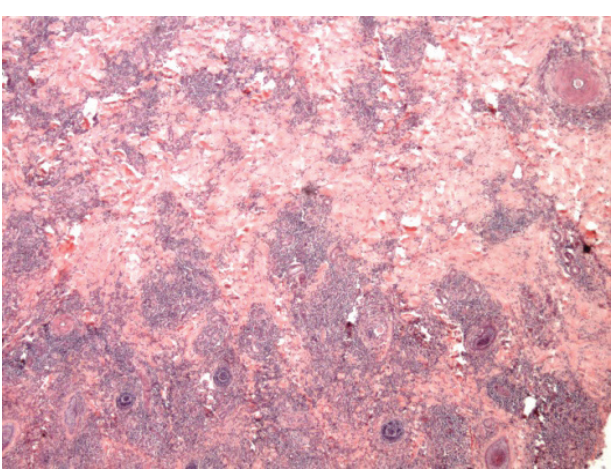

(a)

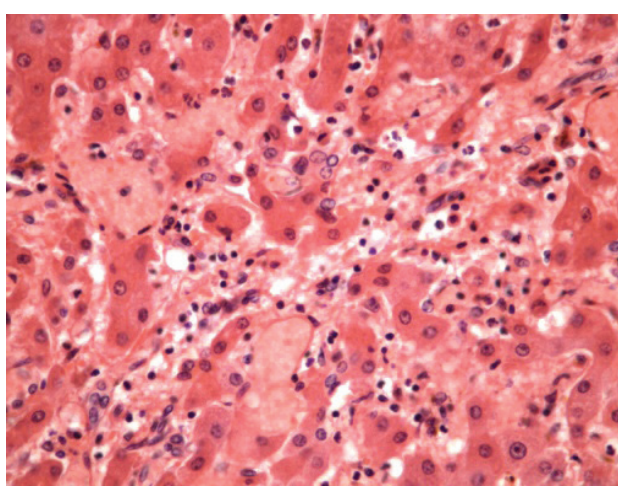

(c)

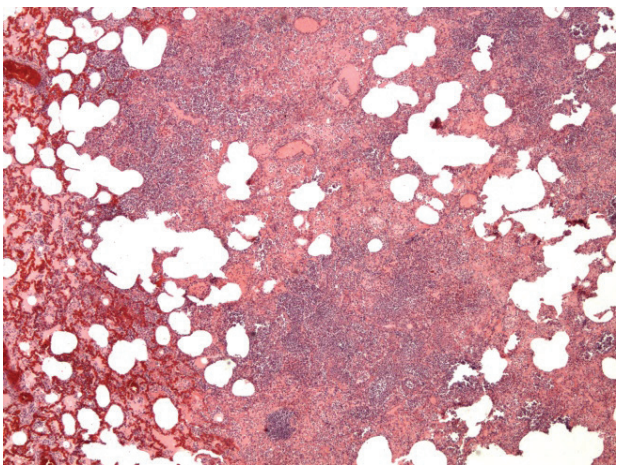

(e)

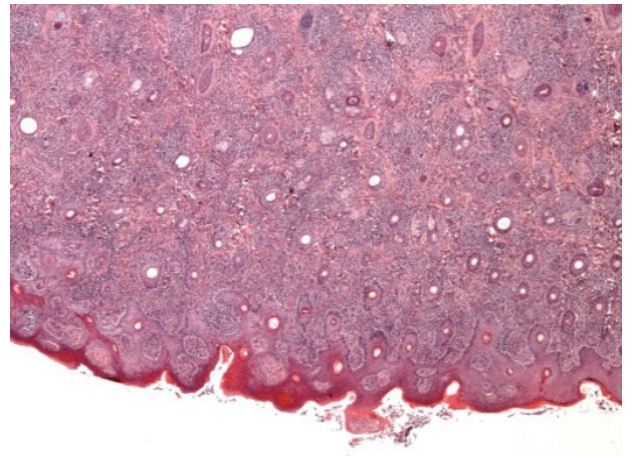

(b)

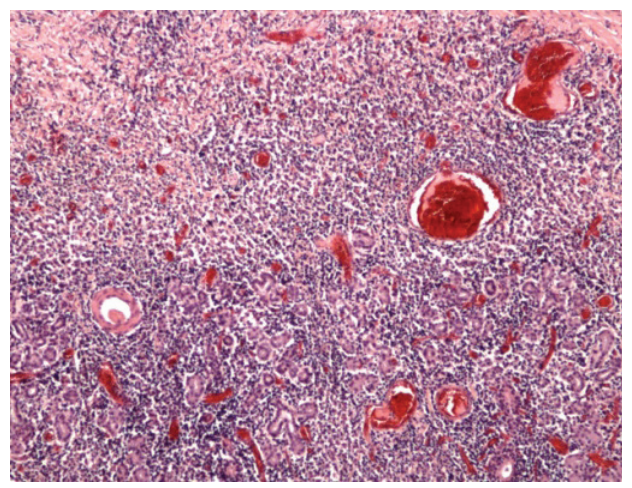

(d)

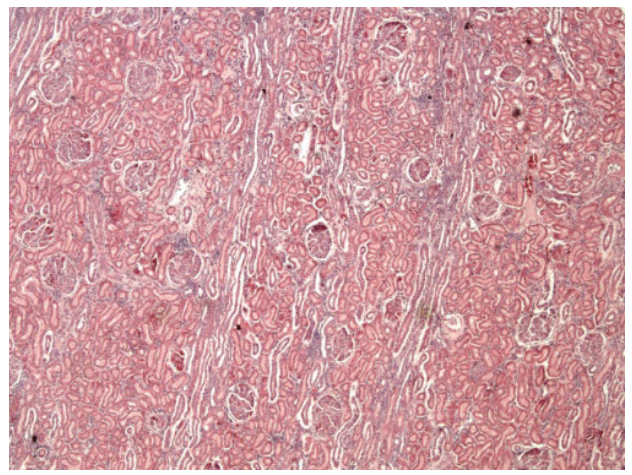

(f)

Figura 2 - Galeria de microfotografias: "Hallazgos histopatológicos en linfoma equino", mostrando varios de los cortes efectuados a diferentes aumentos, donde ambos patólogos involucrados en el diagnóstico señalan la presencia de un abundante infiltrado mononuclear compuesto por linfocitos, bistiocitos y algunas células plasmáticas: (a) Piel que presenta infiltrado predominantemente mononuclear a 4X, (b) Piel con estructuras de apariencia nodular que llegan a ser confluentes, (c) Epitelio nasal presentando infiltrado mononuclear a 20X, (d) Higado con inflltrado, (e) Pulmón, neumonía $4 X$ y (f) Riñón con nefritis intersticial crónica. Fotografías por el Laboratorio de Patologia, Escuela de Medicina Veterinaria, Universidad Nacional, Costa Rica. 
caballos afectados por linfoma (Garber et al., 1994; Janvier et al. 2016). Estos exámenes deberían efectuarse en pacientes con signos clínicos de obstrucción cranial de vena cava en los cuales se detecta efusión pleural o cuando se sospecha presencia de linfosarcoma toráxico (Garber et al., 1994).

\section{Recomendaciones}

1) Debido a que los problemas de piel están entre los dos primeros grupos de patologías afectando caballos es importante incluir este linfoma en el diagnóstico diferencial. 2) Ante la aparición en caballos, de lesiones ulcerativas crónicas y nódulos en varios sitios de la piel y/o mucosas, sumado a pérdida de condición corporal con algunos de los signos clínicos aquí descritos, se recomienda tomar idealmente biopsias por excisión y enviarlas al Laboratorio de Patología para el correcto diagnóstico. 3) Cuando aplique, usar radiografía y/o ultrasonido como complemento diagnóstico para evaluar el avance de las masas neoplásicas.

\section{Referencias}

Bacci, B., Stent, A.W., Walmsley, E.A. (2020). Equine Intestinal Lymphoma: Clinical-Pathological Features, Immunophenotype and Survival. Veterinary Pathology, 57(3): 369-376. https://doi. org/10.1177/0300985820906889.

de Bruijn, C.M., Veenman, J.N., Rutten, E.T., van Nieuwstadt, R.A. \& van den Ingh, T.S. (2007). Clinical, histopathological and inmunophenotypical findings in five horses with cutaneous malignant lymphoma. Research Veterinary Science, 83(1): 63-72. https://doi.org/10.1016/j.rvsc.2006.10.015.

de Clercq, D., van Loon, G., Lefère, L., Deprez, P. (2004). Ultrasound-guided biopsy as a diagnostic aid in three horses with a cranial mediastinal lymphosarcoma. Veterinary Record, 5;154(23):722-6. https:// doi.org/10.1136/vr.154.23.722.

Duran, M.C., Starrak, G., Dickinson, R. \& Montgomery, J. (2016). Peritoneal fluid immunocytochemistry used for the diagnosis of a possible case of equine gastrointestinal B-cell lymphoma. Canadian Veterinary Journal, 57(6): 601-604. PMID: 27247458; PMCID: PMC4866663.

Durham, A.C., Pillitteri, C.A., San Myint, M. \& Valli, V.E. (2013). Two hundred three cases of equine lymphoma classified according to the World Health Organization (WHO) classification criteria. Veterinary Pathology, 50(1): 86-93. https://doi.org/10.1177/0300985812451603.

Garber, J. L., Reef , V. B., Reimer, J. M. (1994). Sonographic findings in horses with mediastinal lymphosarcoma: 13 cases (1985-1992). Journal American Veterinary Medical Association, 205(10):14326. PMID: 7698925

Henson, K.L., Alleman, A.R., Kelley, L.C. \& Mahaffey, E.A. (2000). Immunohistochemical characterization of estrogen and progesterone receptors in lymphoma of Horses. Veterinary Clinical Pathology, 29(2): 40-46. https://doi.org/10.1111/j.1939-165X.2000.tb00396.x.

Janvier, V., Evrard, L., Cerri, S., Gougnard, A., Busoni,V. (2016). Ultrasonographic findings in 13 horses with lymphoma. Veterinary Radiology Ultrasound, 57(1):65-74. https://doi.org/10.1111/vru.12302. 
Kelley, L.C \& Mahaffey, E.A. (1998). Equine malignant lymphomas: morphologic and immunohistochemical classification. Veterinary Pathology, 35(4): 241-252. https://doi.org/10.1177/030098589803500402.

Knottenbelt, D.C., Patterson-Kane, J.C. \& Snalune, K.L. (2015). Clinical Equine Oncology. Elsevier. Edinburg. p.342- 362.

Knowles, E.J., Tremaine, W. H., Pearson, G. R., Mair, T.S. (2016). A database survey of equine tumours in the United Kingdom. Equine Veterinary Journal, 48(3):280-4. https://doi.org/10.1111/evj.12421

Larsdotter, S., Nostell, K., von Euler, H. (2015). Serum thymidine kinase activity in clinically healthy and diseased horses: a potential marker for lymphoma. Veterinary Journal, 205(2):313-316. https://doi. org/10.1016/j.tvil.2015.01.019.

Littlewood, J.D., Whitwell, K.E. \& Day, M.J. (1995). Equine cutaneous lymphoma: a case report. Veterinary Dermatology, 6(2):105-111. https://doi.org/10.1111/j.1365-3164.1995.tb00051.x

Luethy, D., Frimberger, A.E., Bedenice, D., Byrne, B.S., Groover, E.S., Gardner, R.B., Lewis, T., MacDonald, V.S., Proctor-Brown, L., Tomlinson, J.E., Rassnick, K.M. \& Johnson, A.L. (2019). Retrospective evaluation of clinical outcome after chemotherapy for lymphoma in 15 equids (1991-2017). Journal Veterinary Internal Medicine, 33(2): 953-960. https://doi.org/10.1111/jvim.15411

Matsuda, K., Shimada, T., Kawamura, Y., Sakaguchi, K., Tagami, M., Taniyama, H.J. (2013.) Jejunal intussusception associated with lymphoma in a horse. Veterinary Medicine Science, 75(9):1253-6. https://doi.org/10.1292/jvms.13-0060.

Meisel, R., Zibert, A., Laryea, M., Gobel, U., Daubener, W. \& Dilloo, D. (2004). Human bone marrow stromal cells inhibit allogenic T-cell responses by indoleamine 2,3-dioxygenase-mediated tryptophan degradation. Blood, 103(12): 4619-21. https://doi.org/10.1182/blood-2003-11-3909.

Meyer, J., Delay, J. \& Bienzle, D. (2006). Clinical, laboratory, and histopathologic features of equine lymphoma. Veterinary Pathology, 43(6): 914-924. https://doi.org/10.1354/vp.43-6-914.

Miglio, A., Morelli, C., Gialletti, R., Laureti, E., Sforna, M., Marenzoni, M.L. \& Antognoni, M.T. (2019). Clinical and immunophenotypic findings in 4 forms of equine lymphoma. Canadian Veterinary Journal, 60(1): 33-40. PMID: 30651648; PMCID: PMC6294024.

Miller, C.A., Durham, A.C., Schaffer, P.A., Ehrhart, E.J., Powers, B.E. \& Duncan, C.G. (2015). Classification and clinical features in 88 cases of equine cutaneous lymphoma. Journal Veterinary Diagnostic Investigation, 27(1): 86-91. https://doi.org/10.1177/1040638714561653.

Muñoz, A., Riber, C., Trigo, P., Castejón F. (2009). Hematopoietic Neoplasias in Horses: Myeloproliferative and Lymphoproliferative Disorders. Journal Equine Science, 20(4): 59-72. https://doi.org/10.1294/ jes.20.59

Murphy, C.J., Lavoie, J.P., Groff, J., Hacker, D., Pryor, P. \& Bellhorn, R.W. (1989). Bilateral eyelid swelling attributable to lymphosarcoma in a horse. Journal American Veterinary Medical Association, 194(7):939-942. PMID: 2703429 
Rebhun, W.C. \& Del Piero, F. (1998). Ocular lesions in horses with lymphosarcoma: 21 cases (1977-1997). Journal American Veterinary Medical Association, 212(6): 852-854. PMID: 9530426

Scott, D.W \& Miller, W.H. (2010). Equine dermatology, $2^{\text {nd }}$ Edition. Saunders, St. Louis.

Taintor, J. \& Schleis, S. (2011). Equine lymphoma. Equine Veterinary Education, 23(4): 205-213. https://doi. org/10.1111/j.2042-3292.2010.00200.x

Valli, V.E., Jacobs, R.M. \& Parodi, A.L. (2002). Histological classification of hematopoietic tumors of domestic animals. Armed Forces Institute of Pathology, Washington DC.

Valli, V.E. (2007). Hematopoietic system. In Jubb, K.V., Kennedy, P.C. \& Palmer, N. (Eds.). Pathology of Domestic Animals. Saunders, New York. p.150-152. 